Société d'histoire de la révolution de 1848 et des

révolutions du XIXe siècle

56 | 2018

Un autre $\mathrm{XIX}^{\mathrm{e}}$ siècle : I'Inde sous domination coloniale

\title{
Pour une histoire connectée des États princiers indiens
}

\section{Teresa Segura-Garcia}

\section{OpenEdition}

\section{Journals}

Édition électronique

URL : http://journals.openedition.org/rh19/5523

DOl : $10.4000 /$ rh 19.5523

ISSN : $1777-5329$

\section{Éditeur}

La Société de 1848

\section{Édition imprimée}

Date de publication : 15 octobre 2018

Pagination : 132-134

ISSN : 1265-1354

\section{Référence électronique}

Teresa Segura-Garcia, « Pour une histoire connectée des États princiers indiens », Revue d'histoire du XIXe siècle [En ligne], 56 | 2018, mis en ligne le 15 octobre 2020, consulté le 05 janvier 2021. URL http://journals.openedition.org/rh19/5523 ; DOI : https://doi.org/10.4000/rh19.5523 


\section{TERESA SEGURA-GARCIA \\ Pour une histoire connectée des États princiers indiens}

Au cours des deux dernières décennies, les historiens de l'Asie du Sud au $\mathrm{XIX}^{\mathrm{e}}$ siècle ont retracé les liens de l'Inde britannique avec sa métropole, avec les autres colonies et avec d'autres empires - une approche qui reflète la montée de la «nouvelle histoire impériale», ainsi que le «tournant global» dans les sciences humaines. Dans leur empressement à penser au-delà des frontières nationales, les spécialistes de l'Asie du Sud ont en fait été à la pointe de ces deux historiographies ${ }^{15}$. Cependant, leurs explorations sophistiquées des connexions globales de l'Inde britannique n'ont généralement pas inclus l'Inde princière - qui, à travers plus de 500 États princiers, constituait les deux cinquièmes du sous-continent et qu'habitait un tiers de sa population. Après leur accès aux États nouvellement créés de l'Inde et du Pakistan, les États princiers ont été rétroactivement considérés comme des impasses dans l'inévitable marche vers la décolonisation, ce qui en a fait des sujets peu attrayants d'enquête historique.

Il y eut néanmoins quelques exceptions. Dès les années 1970, certains historiens ont plaidé pour le rôle central de ces États dans le maintien de l'Inde britannique ${ }^{16}$. Au cours des décennies suivantes, une série de contributions remarquables les ont ramenés de la périphérie à une position plus centrale au sein de l'historiographie ${ }^{17}$. Cependant, ces entités étaient encore souvent présentées comme des espaces politiques conservateurs avec des «systèmes de valeurs de l'ancien monde sans complexité ${ }^{18}$. Perçus comme des marges traditionnelles résolument opposées à la modernité coloniale de l'Inde britannique, ils paraissaient très éloignés des processus globaux qui intéressaient les historiens de l'Inde britannique et du grand projet impérial britannique au sens large.

15. Sugata Bose, $A$ hundred horizons. The Indian Ocean in the age of global empire, Cambridge (Mass.), Harvard University Press, 2006; Tim Harper et Sunil S. Amrith (eds), Sites of Asian interaction. Ideas, networks and mobility, New Delhi, Cambridge University Press, 2014; Sunil S. Amrith, Crossing the Bay of Bengal. The furies of nature and the fortunes of migrants, Cambridge (Mass.), Harvard University Press, 2013; Durba Ghosh et Dane Kennedy (eds), Decentring empire. Britain, India and the transcolonial world, New Delhi, Orient Longman, 2006; Ashwini Tambe et Harald Fischer-Tiné (eds), The limits of British colonial control in South Asia. Spaces of disorder in the Indian Ocean region, London, Routledge, 2009; Thomas R. Metcalf, Imperial connections. India in the Indian Ocean arena, Berkeley, University of California Press, 2007; Claude Markovits, Jacques Pouchepadass et Sanjay Subrahmanyam (eds), Society and circulation. Mobile people and itinerant cultures in South Asia, 1750-1950, London, Anthem Press, 2006.

16. Robin Jeffrey (ed.), People, princes and paramount power. Society and politics in the Indian princely states, New Delhi, Oxford University Press, 1978.

17. Manu Bhagavan, Sovereign spheres. Princes, education and empire in colonial India, New Delhi, Oxford University Press, 2003; Ian Copland, The British Raj and the Indian princes. Paramountcy in western India, 1857-1930, London, Sangam, 1982; Waltraud Ernst et Biswamoy Pati (eds), India's princely states. People, princes and colonialism, London, Routledge, 2007; Barbara Ramusack, The Indian princes and their states, Cambridge, Cambridge University Press, 2004.

18. Ian Copland, The British Raj and..., op. cit., p. 87. 
Cependant, depuis le début des années 2000, une série de nouvelles approches méthodologiques, théoriques et disciplinaires appliqués à l'étude des États princiers a progressivement émergé. Ces travaux récents ont réévalué l'engagement des dirigeants indiens dans les réformes sociales et politiques, soulignant leur capacité à articuler des projets de modernité alternatifs et non coloniaux pour l'Inde ${ }^{19}$. Tout en remettant en question de vieilles hypothèses et en proposant de nouvelles façons de comprendre ces États, certains de ces nouveaux travaux ont commencé à examiner leurs connexions globales. Cette nouvelle histoire des États princiers est représentée par Eric Beverley, Kate Boehme, Satadru Sen et Chitralekha Zutshi. Ils ont respectivement étudié Hyderabad, les États d'Inde occidentale, Nawanagar et le Cachemire dans leurs milieux plus larges, envisageant les aspects tant politiques que religieux, commerciaux, sociaux, linguistiques et sportiff ${ }^{20}$. La contribution de Beverley est particulièrement remarquable : il a accordé moins d'importance à la politique interne d'Hyderabad, déplaçant l'attention de la cour vers la plus large dynamique de l'État. Cela lui a permis de se concentrer sur les contacts d'Hyderabad au-delà du sous-continent, retraçant les réseaux musulmans de collaboration intellectuelle internationale - «l'internationalisme musulman» - qui animaient la politique d'État et représentaient une alternative à la modernité coloniale britannique.

Ma propre recherche a retracé les liens globaux des États princiers à travers une étude poussée de la longue carrière politique nomadisante du maharaja Sayaji Rao Gaekwad III de Baroda, qui a dirigé le principal État d'Inde occidentale de 1875 à $1939^{21}$. Au cours de ses six décennies de règne, le maharaja de Baroda a établi et maintenu un large éventail de contacts politiques avec des individus et institutions dans l'ouest de l'Inde, le reste du sous-continent et dans le monde. Certaines de ces relations ont été forgées à travers ses voyages : au cours de ses vingt-huit tournées internationales, le

19. Pour quelques-unes des contributions les plus notables, $c f$. Manu Bhagavan, Sovereign spheres. Princes..., op. cit.; Julie E. Hughes, Animal kingdoms. Hunting, the environment and power in the Indian princely states, Cambridge (Mass.), Harvard University Press, 2013; Aya Ikegame, Princely India re-imagined. A historical anthropology of Mysore from 1799 to the present, London, Routledge, 2012; Janaki Nair, Mysore modern. Rethinking the region under princely rule, Minneapolis, University of Minnesota Press, 2011; Norbert Peabody, Hindu kingship and polity in precolonial India, Cambridge, Cambridge University Press, 2003.

20. Eric Beverley, Hyderabad, British India and the world, Cambridge, Cambridge University Press, 2015; Kate Boehme, Commercial networks and the making of a colonial bourgeoisie in western India, 1845-1870, Thèse d'histoire sous la direction de David Washbrook, University of Cambridge, 2015, et "Smuggling India. Deconstructing western India’s illicit export trade, 1818-1870", Journal of the Royal Asiatic Society, volume 25, no 4, 2015, p. 685-704; Chitralekha Zutshi, Languages of belonging. Islam, regional identity and the making of Kashmir, New Delhi, Permanent Black, 2003, and Kashmir's contested pasts. Narratives, sacred geographies and the historical imagination, New Delhi, Oxford University Press, 2014; Satadru Sen, Migrant races. Empire, identity and K. S. Ranjitsinhji, Manchester, Manchester University Press, 2004.

21. Teresa Segura-Garcia, "The lens and the maharaja. The photographic remaking of Indian kingship in the late colonial period", in Marcus Banks et Annamaria Motrescu-Mayes (eds), Visual histories of South Asia, New Delhi, Primus Books, 2018, p. 115-131; et Baroda, the British empire and the world, c. 1875-1939, Thèse d'histoire sous la direction de C. A. Bayly, University of Cambridge, 2015. 
dirigeant a visité de long en large l'Europe, l'Amérique du Nord, l'Afrique et l'Asie de l'Est. Il a profité de ces tournées pour rencontrer et apporter un soutien financier à des éléments politiquement actifs de la diaspora indienne, du leader du Congrès national indien basé à Londres, Dadabhai Naoroji, aux figures révolutionnaires en exil telles que Bhikaji Cama et Shyamji Krishnavarma. Loin d'être une île de tradition, Baroda était connectée aux réseaux de personnes, d'idées et de ressources qui soudaient l'Empire britannique tout en contribuant à sa disparition potentielle. Bien que Baroda ne soit pas représentatif des États princiers dans leur ensemble, certaines de ses caractéristiques politiques sont présentes ailleurs. Les dirigeants d'autres États de l'Inde occidentale - en particulier Kolhapur - se sont également engagés dans des politiques anticoloniales au-delà de leurs frontières.

De récentes critiques ont mis en évidence les dangers du «tournant global» en histoire, qui consiste à présenter des connexions anciennes comme quantitativement et qualitativement continues, ignorant les hauts et les bas et ne formulant pas de cadres explicatifs complets ${ }^{22}$. Alors que le tournant global a peut-être atteint ce que David A. Bell a qualifié de "point de rendements décroissants" dans d'autres domaines, il conserve sa valeur pour l'étude des États princiers indiens ${ }^{23}$. L'approche des États à travers le tournant global nous permet de les considérer comme une partie constitutive de l'Inde et de l'Empire britannique, soulignant leur position ambivalente en tant que contreforts du pouvoir impérial et agents de décolonisation. Cette enquête, qui réétalonne notre compréhension des limites de l'Empire britannique, peut faire de même pour d'autres projets impériaux contemporains, où les formes locales de royauté et d'autorité coexistaient avec les régimes coloniaux.

\section{Teresa Segura-Garcia est chercheuse Juan de la Cierva à l'université Pompeu Fabra, à Barcelone}

\section{IAN J. KERR \\ Technologie et transfert de technologie dans l'Empire britannique en Inde}

$\mathrm{Au} \mathrm{XIX}^{\mathrm{e}}$ siècle, les partisans de la présence impériale britannique sur le sous-continent indien et ailleurs pensaient que la technologie permettrait de renforcer l'autorité coloniale et de favoriser les intérêts de la Grande-Bre-

22. Samuel Moyn et Andrew Sartori, "Approaches to global intellectual history”, in Samuel Moyn et Andrew Sartori (eds), Global intellectual history, New York, Columbia University Press, 2015, p. 3-32 (p. 21-23).

23. David A. Bell, "This is what happens when historians overuse the idea of the network", New Republic, <https://newrepublic.com/article/114709/world-connecting-reviewed-historiansoveruse-network-metaphor> [consulté le $1^{\text {er }}$ décembre 2017] 